\title{
A Dielectrophoresis-based Array Cytometer
}

\author{
Joel Voldman*, Mehmet Toner**, Martha L. Gray*, and Martin A. Schmidt* \\ * Massachusetts Institute of Technology, Cambridge, MA 02139 USA \\ ** Center for Engineering in Medicine, Massachusetts General Hospital, Boston, MA 02114
}

\section{SUMMARY}

We present the initial results of a cytometer that uses an array of extruded high-performance dielectrophoretic traps to capture, hold, and selectively release multiple single bioparticles. We have fabricated small arrays of traps and demonstrated single-bead and single-cell trapping, electrically switched bead release from single traps, fluorescence microscopy of trapped viable single cells, and quantitatively predicted trap strength.

Keywords: dielectrophoresis, cell traps, single-cell analysis

\section{INTRODUCTION}

Dielectrophoresis (DEP) - the action of polarizable particles in non-uniform electric fields - can be used to produce stable traps for bioparticles [1-3] as well as provide cell separation based upon size or electrical properties $[4,5]$.

We are developing a planar cytometer than is comprised of a two-dimensional array of electrically switchable DEP traps, each of which will hold a single cell. Such a cytometer can be used to perform parallel single-cell assays. In order to design the individual traps for this cytometer, we have taken advantage of quantitative modeling tools that we previously developed to predict the holding strength of DEP-based traps [6, 7].

\section{TRAP DESIGN}

The traps for the cytometer needed to be easily arrayed, batch fabricated, and exhibit strong holding against flow, since that is the destabilizing force. We have chosen the commonly used quadrupole trap excited with alternating voltages, but made the electrodes from extruded posts (Fig. 1). There are several reasons for this. First, the weakness of planar quadrupole traps is due to the 1) decay of the fields away from the substrate and 2) the increase in levitation height as the voltage is increased, which exposes the particle to higher drag [7]. These effects serve to limit the strength of such traps to $\sim 1 \mathrm{pN}$ [7]. Second, extruded electrodes, although they can be serially processed [8], are amenable to batch fabrication. Third, extruded quadrupoles have the capacity to provide strong confinement with only four electrodes.

We used our modeling tools to determine how to vary the dimensions of the extruded quadrupole to give strong holding against flow while minimizing the electric-field loading of the cells and the shear stress on the cells. Further details will be given in [9].

This led us to the asymmetric trapezoidal arrangement of electrodes, rather than a square quadrupole. Such an
(A)

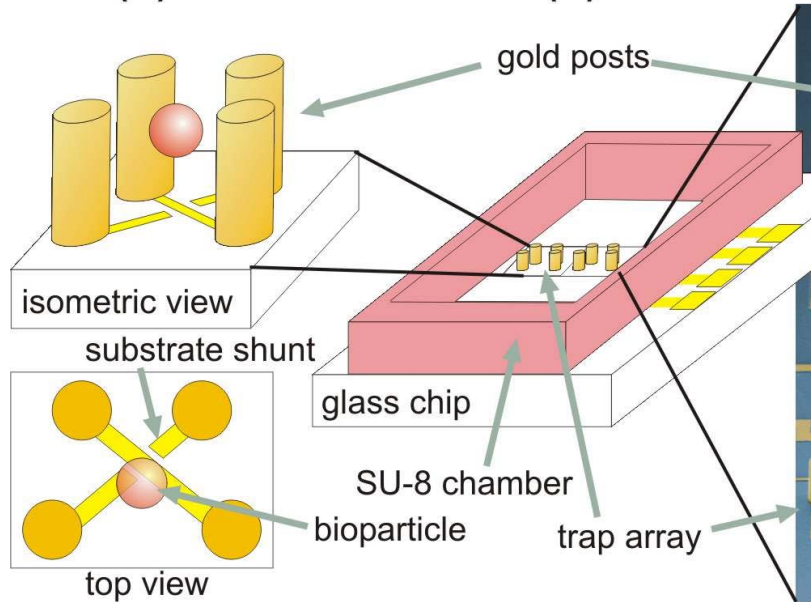

(C)

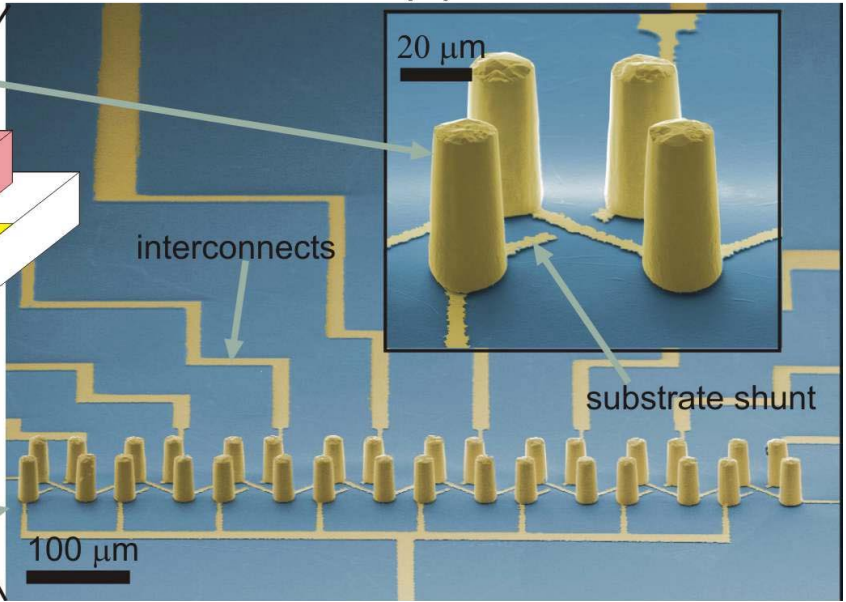

Fig. 1: Schematic of trap array. (A) Two views of a single trap, illustrating the trapezoidal placement of the gold posts and a bioparticle suspended in the trap. (B) The trap is one of an array of traps. (C) SEMs of a 1x8 array of traps along with an exploded view of one trap. 
arrangement makes it easier to load particles into the trap without sacrificing trap strength.

Furthermore, we were able to use the modeling environment to refine the traps. One issue was that to use only one layer of metal, we needed to route an interconnect through the middle of the trap. We determined that the addition of substrate shunts (Fig. 1A,C) gave much better holding than just using a single substrate interconnect. In addition, we determined that by manipulating the potential on only one of the four electrodes we could eject particles from the trap and thus effect sorting.

\section{ARRAY FABRICATION}

The fabrication process starts with Pyrex wafers upon which we deposit a Ti/Au layer (Fig. 2A). We then pattern etch the Au layer in a potassium iodide solution (Gold Etchant Type-TFA, Transene, Danvers, MA) to define the substrate interconnects (Fig. 2B). We use such a process, instead of liftoff, because it allows us to pattern the Au layer while keeping a continuous Ti layer on the wafer. We will use this Ti layer as an electrical connection layer during subsequent electroplating.

The electrodes are made from gold posts electroplated into a $60-\mu \mathrm{m}$-thick SU-8 mold (Fig. 2C-D). The keys to patterning the SU-8 for this step are to maximize adhesion and minimize edge bead. To enhance adhesion, we treat the wafer to a 10-min Nanostrip clean, a $1-\mathrm{hr}$ dehydration bake at $200^{\circ} \mathrm{C}$, and a $20-\mathrm{min}$ UV-ozone descum. After coating, we let the SU-8 relax on the wafer at room temperature for 30-min and then ramp the hotplate to the prebake temperature $\left(105^{\circ} \mathrm{C}\right)$ to minimize any thermal shock. After prebake, we lightly run a PGMEA-coated swab around the wafer to reduce the edge-bead and then perform a short bake to evaporate the solvent $\left(20-\min @ 50^{\circ} \mathrm{C}\right)$.

After the SU-8 photo step, we electroplate the gold

(A)
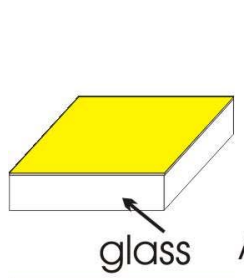
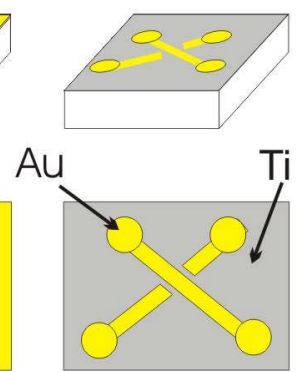

(C)

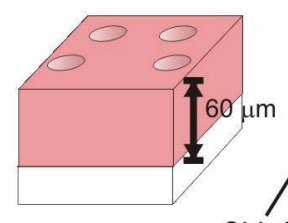

SU-8

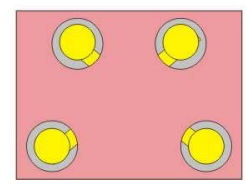

posts with a AuCN bath (Orotemp 24, Technic Inc., Anaheim, CA). We descum the SU-8 in an asher immediately prior to this step, which aids electroplating initiation. We visually endpointed the electroplating before the posts reached the top of the SU-8.

Removing SU-8 molds is often a difficult process. We accomplished this step by 1) reducing baking times and temperatures during the photolithography step, 2) keeping the time that the SU-8 is actually on the wafer to $<3$ days (D. McIntosh, Ashland ACT, personal comm.), and 3) using a multi-step strip sequence. The strip involves 1) a DMSO-based photoresist stripper (Ashland Chemical Co., Pueblo, CO), followed by 2) an ash to remove SU-8 left over from the previous step, and 3) a Nanostrip treatment to give a final clean. This was successful in stripping the SU-8 layer while leaving the electroplated posts intact.

The final steps, after stripping the Ti layer (Fig. 2F), are to make a $150-\mu \mathrm{m}$-thick SU-8 fluidic chamber, dice the wafer, drill fluidic access holes with a diamond drill bit (C. R. Laurence, Los Angeles, CA), and glue a coverslip atop the channel.

\section{PACKAGING AND TEST SETUP}

To provide electrical, optical, and fluidic access to the chip, we used the packaging scheme shown in Fig. 3. The electrical interconnections were made by wirebonding out from the edge of the chip to a ceramic carrier that sits in a ZIF-socket. The ZIF-socket is connected to a printed-circuit board that houses the drive circuits. The fluidic connections are made via the flow path shown in Fig. 3; the flow is brought via PEEK tubing to an aluminum shunt that sits in a cutout in the ZIF socket. The ceramic carrier mates with the aluminum shunt via o-rings. The chip connects to the ceramic carrier with double-sided tape. Optical access is provided through the coverslip.
(D)

(E)
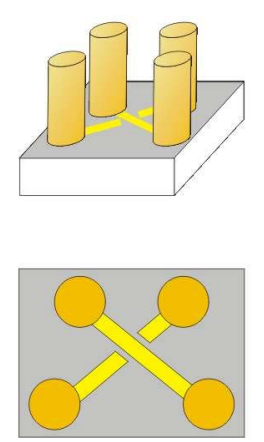

(F)

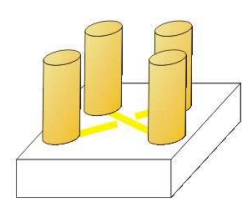

Fig. 2: Fabrication process flow for the extruded traps 


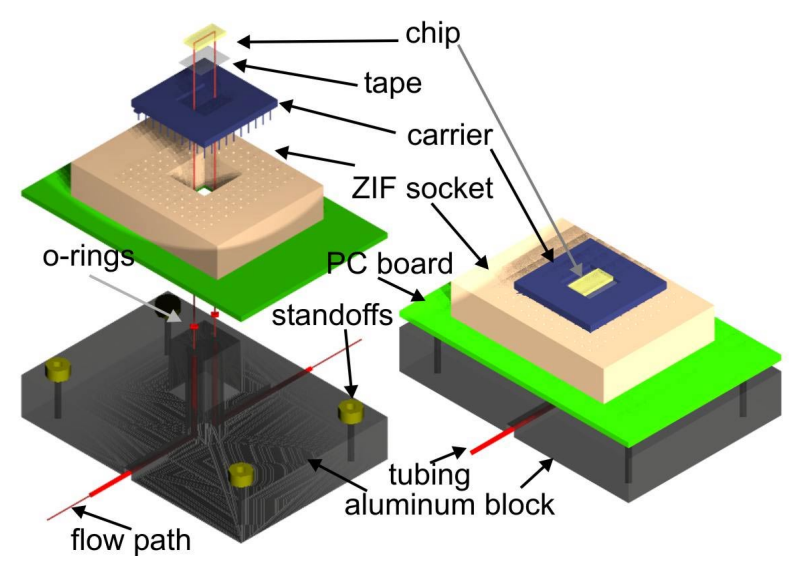

Fig. 3: Packaging scheme

The low-profile assembly sits on an upright fluorescence microscope (Zeiss Universal). Syringe pumps provide flow to the chip while an HP signal generator and electrically switched buffer amplifiers provide electrical signals of up to $5 \mathrm{~V}_{\mathrm{pp}}$ at $20 \mathrm{MHz}$ to the chip. The arrays were excited with AC voltage on one pair of electrodes while other pair was grounded.

\section{EXPERIMENTAL RESULTS}

\section{Materials}

Polystyrene beads were purchased from Bangs Laboratories (Fishers, IN) and suspended in $0.01 \mathrm{~S} / \mathrm{m}$ HBSS solutions with $0.05 \%$ Triton X-100. All bead solutions were refrigerated when not in use. HL-60 cells were maintained in RPMI supplemented with $20 \%$ fetal bovine serum, $1 \%$ L-glutamine and 1\% PenStrep. Immediately prior to assay, cell aliquots were washed $3 \mathrm{x}$ in HBSS and incubated for 30-45 minutes with $5 \mu \mathrm{M}$ calcein-AM (Molecular Probes, Oregon).

\section{Trapping of beads \& cells}

Fig. 4 shows a top-down view of a $1 \times 4$ array of traps with a single $10.0-\mu \mathrm{m}$ bead held in each trap. We can also trap single viable cells in these traps, as shown in

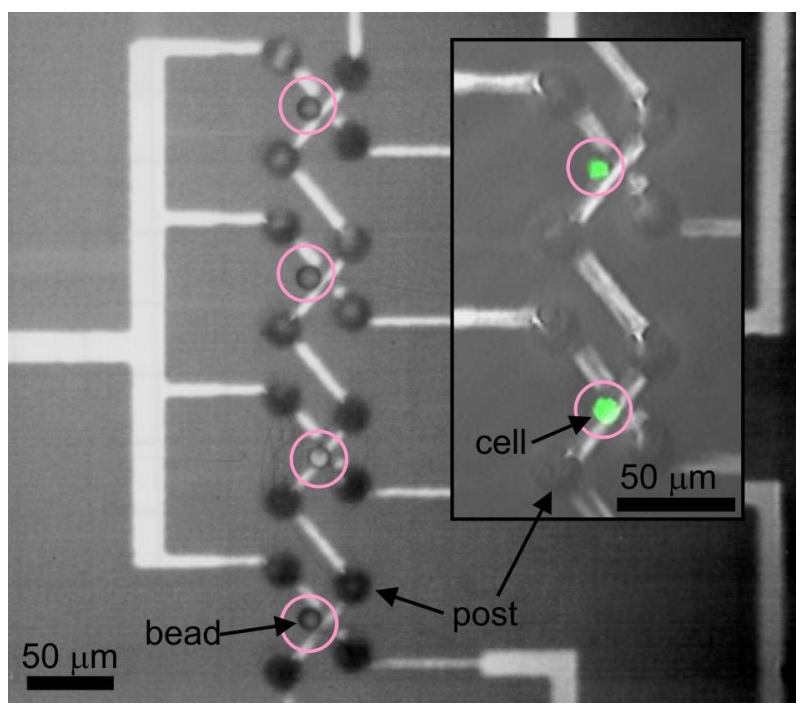

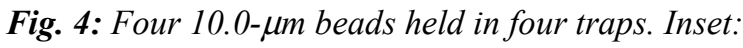
Two trapped calcein-labeled HL-60 cells

the inset of Fig. 4.

One of the requirements of the cytometer is to be able to switch individual traps on and off. We do this by switching the excitation at one of the electrodes from $\mathrm{AC}$ to ground. This disrupts the quadrupolar field and releases the particle. An example of this is shown in Fig. 5. Here we show an array of traps with a single bead held in each of two traps. By applying a flow and selectively turning one trap off we can release one of the beads and hold the other. Thus we can effect sorting of beads held in the traps.

In order to trap one and only one particle per trap, we make use of the fact that a trap is essentially a potential energy well, and that the size of this well varies with the applied flowrate. Specifically, the size of the potential energy well varies inversely with the flowrate, so that one can trap several particles in the trap at low flowrates and none at high enough flowrates. If we set the flowrate in the chamber to $\sim 90 \%$ (determined
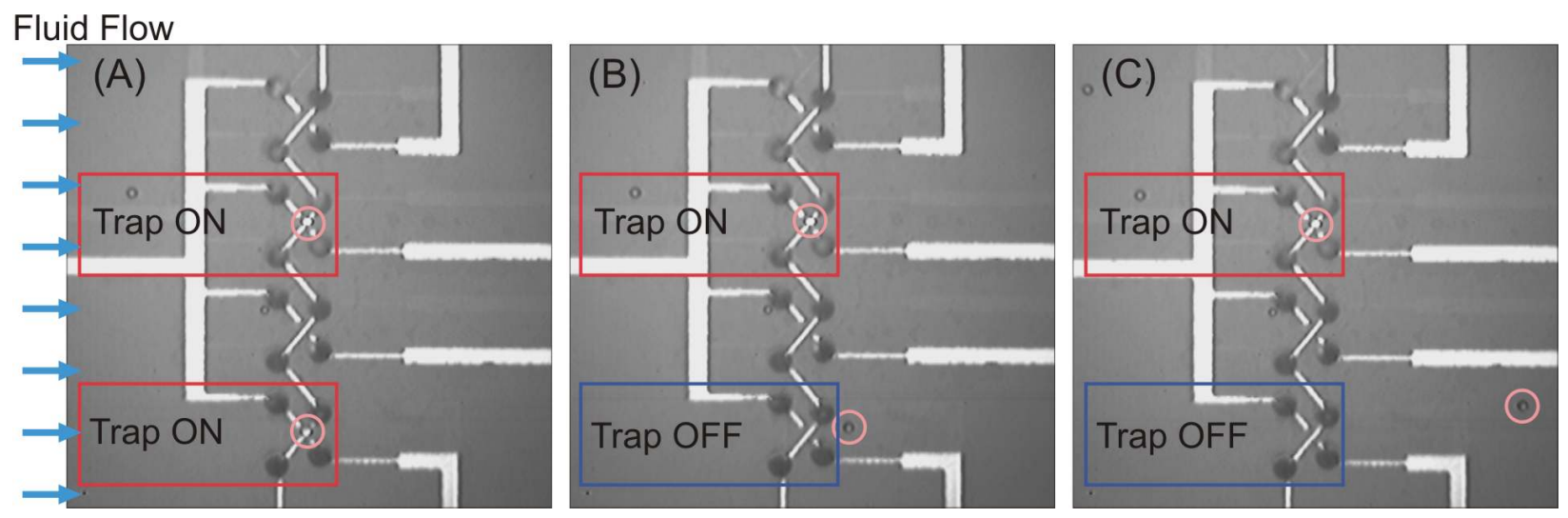

Fig. 5: Selective release of bioparticles under fluid flow. Three time-sequence views of traps with 7.6- $\mu$ m beads in two of the traps. By switching the bottom trap off, we can release its bead \& still hold the bead in the upper trap. 
empirically) of the maximum flowrate that a particle in the trap can sustain, then the size of the potential energy well decreases to the point where only one particle can fit in the trap.

Finally, we have also demonstrated that these traps are as strong as designed. We have been able to match predictions of the holding characteristics (release flowrate versus voltage) for different beads with experimental results (Fig. 6). The extracted holding forces are $\sim 100 \mathrm{pN}$ for 13.2- $\mu \mathrm{m}$ beads at $2 \mathrm{~V}$ [9].

\section{DISCUSSION}

The planar cytometer that we are trying to realize is a highly demanding application because of the need for compact, easily arrayable, and batch-fabricatable traps that exhibit strong holding against flow. The requirement for holding comes from the actual use the cytometer - in order to add reagents and cells to the chamber, the traps must withstand flow.

Our modeling tools give us an avenue towards meeting these design constraints, in that we can perform design iterations in software and predict a priori the performance of a given trap design. They give us insight into the design that would be difficult to come by otherwise. Examples of such insight are the use of a trapezoidal rather than square electrode geometry and the addition of substrate shunts to restore holding when a substrate interconnect passes through the trap.

We have been able to demonstrate all the key elements necessary for this cytometer - holding of beads and cells, selective release of particles in traps, strong holding against flow, and single-particle holding in the traps. The next challenges come from scaling up the

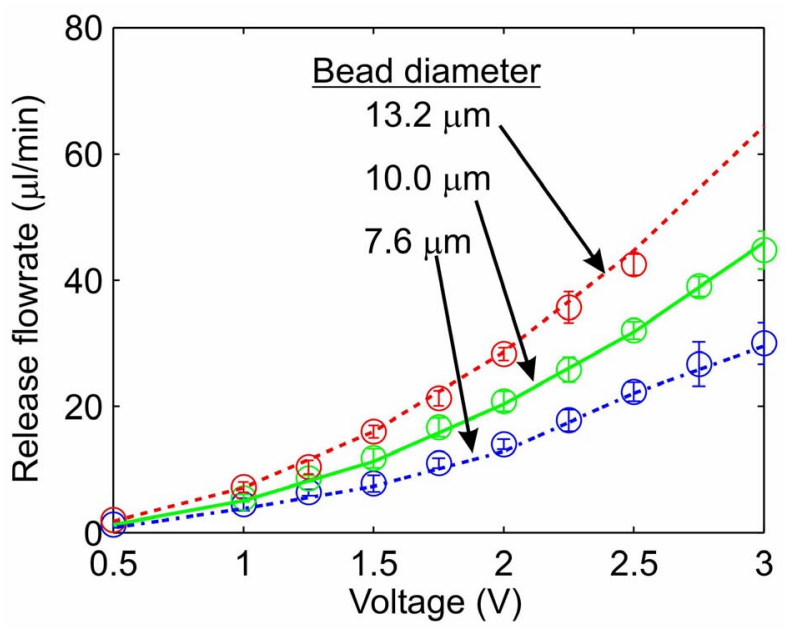

Fig. 6: Comparison of predicted (-) and measured (०) flowrates needed to dislodge beads from the traps array and designing the optics necessary to image it.

\section{CONCLUSIONS}

We have demonstrated an array of dielectrophoretic traps - in an extruded quadrupole configuration - for eventual use in a cytometer for trapping single micronsized bioparticles such as beads and cells. By quantitatively designing the traps, we have been able to attain the high performance necessary for such a demanding application.

\section{ACKNOWLEDGEMENTS}

We would like to thank the Microsystems Technology Laboratories, where the fabrication was performed. This work is supported by the Alliance for Cellular Signaling (NIGMS). Mr. Voldman is supported by a Kodak Research Fellowship.

\section{REFERENCES}

[1] T. Muller, G. Gradl, S. Howitz, S. Shirley, T. Schnelle, and G. Fuhr, "A 3-D microelectrode system for handling and caging single cells and particles," Biosens. Bioelec., 14(3):247, 1999.

[2] T. Schnelle, R. Hagedorn, G. Fuhr, S. Fiedler, and T. Muller, "3-Dimensional electric-field traps for manipulation of cells - calculation and experimental verification," Biochim. Biophys. Acta, 1157(2):127-140, 1993.

[3] N. G. Green and H. Morgan, "Dielectrophoresis of submicrometer latex spheres. 1. Experimental results," J. Phys. Chem. B, 103(1):41-50, 1999.

[4] H. Morgan, M. P. Hughes, and N. G. Green, "Separation of submicron bioparticles by dielectrophoresis," Biophys. J, 77(1):516, 1999

[5] J. Yang, Y. Huang, and P. R. C. Gascoyne, "Cell Separation on Microfabricated Electrodes Using Dielectrophoretic/Gravitational FieldFlow Fractionation," Anal. chem., 71(5):911, 1999.

[6] J. Voldman, R. A. Braff, M. Toner, M. L. Gray, and M. A. Schmidt, "Quantitative design and analysis of single-particle dielectrophoretic traps," presented at Micro Total Analysis Syst. '00, Twente, Netherlands, 431-4, 2000.

[7] J. Voldman, R. A. Braff, M. Toner, M. L. Gray, and M. A. Schmidt, "Holding Forces of Single-Particle Dielectrophoretic Traps," Biophys. J., 80(1):531-41, 2001.

G. Fuhr, C. Reichle, and M. Stuke, "Processing of micro-particles by UV laser irradiation in a field cage," Appl. phys., 69(6):611, 1999.

[9] J. Voldman, M. Toner, M. L. Gray, and M. A. Schmidt, in preparation, 2001. 